\title{
Dynamic Ensembles with Variable Structure and Friction Simulation
}

\author{
I.V. FELDSTEIN * and N.N. KUZMIN \\ Institute of Applied Mathematics, Russian Academy of Sciences, Miusskaya pl. 4, Moscow, Russia
}

(Received 1 February 1998)

\begin{abstract}
The paper presents an approach to the simulation of friction interaction. The model does not use any physical descriptions of the processes in the system, but it has simple physical interpretation. It is based on one qualitative experimental result - the value of first Lyapunov exponent drops with normal load. It is shown that the logistic map could be considered as the simplest model of continuous contact. The generalization of the model (which takes into account the discreteness of the real contact) gives results very similar to the experimental ones. It is in the form of a dynamic ensemble with variable structure (DEVS), which has some interesting properties - particularly bifurcation diagrams.
\end{abstract}

Keywords: Friction simulation, Ensembles of one-dimensional maps, Lyapunov exponent

\section{INTRODUCTION}

The usual way of simulation of friction interaction is based on the methods of mechanics of continuous media (Kragelsky et al., 1982; Leibovich, 1968). Such a description of contact area includes

1. partial differential equations (e.g. in displacements or stresses),

2. constitutive relationships (like state equation Hooke's law for elastic bodies),

3. local conditions of fracture, and

4. conditions of body's interaction (boundary conditions).
Thus for the body which occupies the domain $\Omega$ we have: from 1, the equation of equilibrium (Landau and Lifshitz, 1987)

$$
\rho \ddot{u}_{i}=\sigma_{i j, j} \quad \text { in } \Omega
$$

( $\rho$ - density of material, $\boldsymbol{u}$ - displacement, $\sigma_{i j}-$ components of stress tensor); from 2, the state equation and relationships between the displacements $\left(u_{i}\right)$ and the strain tensor $\left(\varepsilon_{i j}\right)$,

$$
\begin{gathered}
\sigma=F(\varepsilon, \dot{\varepsilon}, \ldots), \\
\varepsilon_{i j}=\frac{1}{2}\left(u_{i, j}+u_{j, i}+u_{k, j} u_{k, i}\right) ;
\end{gathered}
$$

*Corresponding author. Tel.: (095) 250-78-02. E-mail: ilya@feld.msk.ru. 
from 3,

$$
G\left(\sigma_{i j}, \ldots,\right) G^{*}
$$

(the local conditions of fracture and the state equation may be written in some other forms); and from 4 , the set of boundary conditions on $\partial \Omega$.

The complete model should include also the description of chemical, electrical processes and heat transfer, but even in the simplest case the models obtained are too complex to be correctly analyzed. The nonlinearity in constitutive relationships and the arising of new internal bounds make difficult not only analytical but also numerical analysis of the model.

Such a situation is not specific only for the analysis of friction interaction - similar difficulties are usual for a nonlinear system far from equilibrium with many coexisting and interacting processes.

In this paper we attempt to build a model of friction interaction without any physical assumption about the processes in contact area (the physical interpretation is considered as an additional way to prove the model). Instead, some qualitative experimental results are used as a base for the selection of a mathematical object with the similar behavior.

\section{EXPERIMENTAL RESULTS}

In the previous articles (Kuzmin and Feldstein, 1996; 1997) the dynamic characteristics of friction interaction and some aspects of tribosystem simulation were considered. In brief the main results of these investigations are:

1. Friction force $\boldsymbol{F}_{\text {fr }}$ can be divided into two components - dynamic $\left(\boldsymbol{F}_{\mathrm{dyn}}\right)$ and static $\left(\boldsymbol{F}_{\mathrm{st}}\right)$ : $\boldsymbol{F}_{\mathrm{fr}}=\boldsymbol{F}_{\mathrm{dyn}}+\boldsymbol{F}_{\mathrm{st}}$. The characteristic time for the former is $10-100 \mathrm{~ms}$, and for the latter $10 \mathrm{~s}$ and more.

2. First Lyapunov exponent $\lambda_{1}$ estimated from a time series of $\boldsymbol{F}_{\mathrm{dyn}}$ (Wolf et al., 1985) is mainly positive; that is, in the friction interaction there exist chaotic regimes.

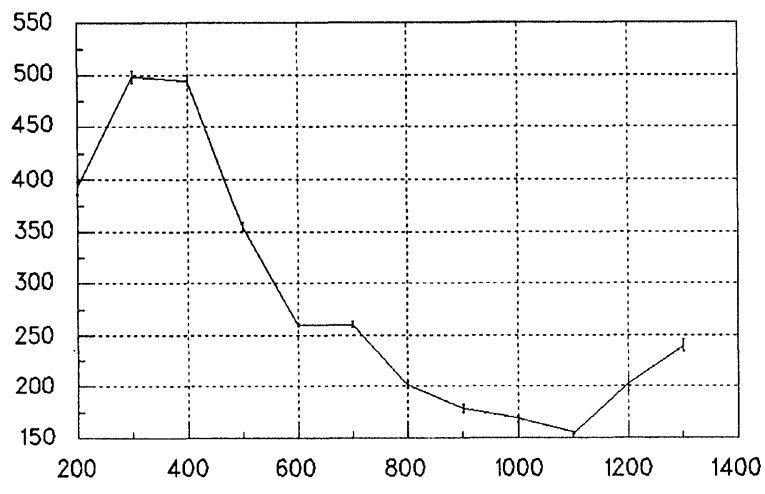

FIGURE 1 The dependence $\lambda_{1}(\boldsymbol{P})$ (from experiment).

3. The value of $\lambda_{1}$ characterizes the current state of tribosystem (it includes the two bodies which are in friction interaction and the media near the contact - lubricants, abrasives, fracture particles, etc.). This result can be obtained by comparing the changes of $\lambda_{1}$ and $\boldsymbol{F}_{\mathrm{st}}$ in operation. The curves $\lambda_{1}(t)$ and $\boldsymbol{F}_{\mathrm{st}}(t)$ are very similar.

4. The value of $\lambda_{1}$ drops monotonically in a wide range of normal load $\boldsymbol{P}$. Figure 1 shows the dependence $\lambda_{1}(\boldsymbol{P})$.

\section{THE MODEL OF CONTINUOUS CONTACT AND PHYSICAL INTERPRETATION}

Let us try to build the model of friction interaction which has the dependence $\lambda_{1}(\boldsymbol{P})$ like the experimental one.

Find first the mathematical object which has the monotone dependence of $\lambda_{1}$ on the parameter. Consider as an example the logistic map

$$
x_{i+1}=\mu x_{i}\left(1-x_{i}\right) .
$$

This map is well known in nonlinear dynamics. Figure 2 shows $\lambda_{1}(\mu)$. This dependence is very irregular, but roughly we can take that its envelope grows monotonically with $\mu$. (Experiment cannot give infinite resolution by parameters, that is why we do not take into account the fine structure of the dependence.) 


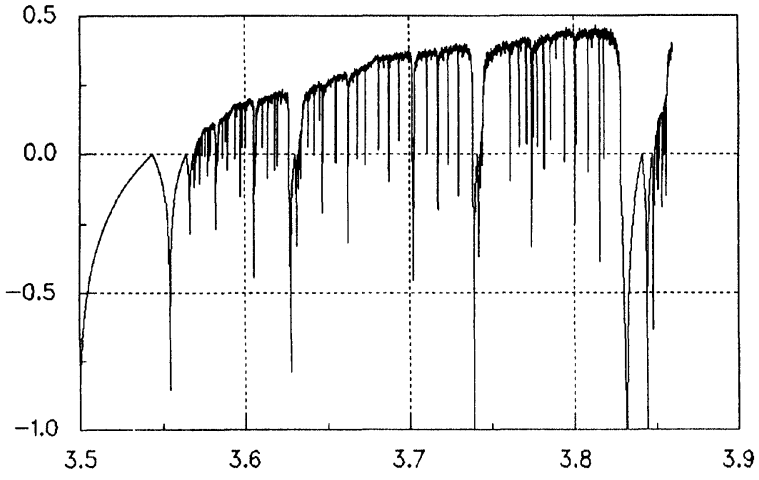

FIGURE 2 The dependence $\lambda_{1}(\mu)$ for logistic map.

In some sense, to build the model it is enough to postulate that $\mu$ drops monotonically with the load $\boldsymbol{P}$ and the variable $\boldsymbol{x}$ is proportional to friction force, but for an understanding of the model structure and its parameters it would be better to offer a physical interpretation.

Suppose that

1. all processes of energy transformation take place in near-surface layer $\mathbf{A}$ of contacting body (see Fig. 3(a));

2. layer $\mathbf{A}$ has a maximal energy capacity $E^{*}$ (if $E>E^{*}$ the layer is destroyed instantly and in next time step the underlying layer $\mathbf{A}^{\prime}$ is considered as A - see Fig. 3(b));

3. the energy of layer $\mathbf{A}$ in the next time step is proportional to the current friction coefficient $k_{i}$ and to the "free capacity" $E^{*}-E_{i}: E_{i+1}=$ $a k_{i}\left(E^{*}-E_{i}\right)$. Then taking into account that $E=$ $\boldsymbol{F}_{\mathrm{fr}} l=k \boldsymbol{P} v t$, where $k$ is the friction coefficient, $\boldsymbol{P}$ the normal load, $v$ the sliding velocity, and $t$ the physical time between two subsequent time steps, we can obtain

$$
x_{i+1}=\mu x_{i}\left(1-x_{i}\right)
$$

where $x_{i}$ is proportional to $k_{i}$, and $\mu$ - to $1 / \boldsymbol{P}$.

Thus there is respectively simple physical interpretation of the model which has a behavior qualitatively similar to that observed in a number of experiments.

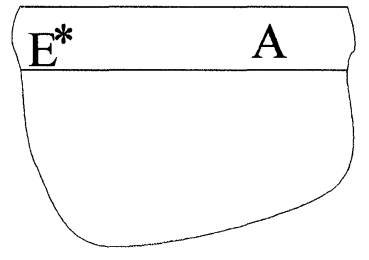

(a)

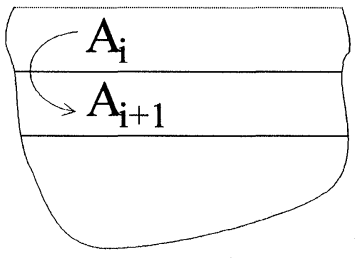

(b)
FIGURE 3 Scheme of near-surface layer.

\section{THE CASE OF DISCRETE CONTACT - GENERALIZATION OF THE MODEL}

The model presented above is valid in the case of continuous contact, whereas real contacts are always discrete - they consist of multiple contact spots. The integral area of contact grows with the normal load.

To generalize the model of continuous contact let us consider an ensemble of $N$ logistic maps. Each map describes the part of nominal contact area. Such a part is in the contact with the probability $q$ and out of it with the probability $1-q$. Thus on the $i$ th step the dynamics of $k$ th element is

$x_{i+1}^{k}=\left\{\begin{array}{l}f\left(x_{i}^{k}\right)=\mu x_{i}^{k}\left(1-x_{i}^{k}\right) \text { with probability } q, \\ x_{i}^{k} \quad \text { with probability } 1-q,\end{array}\right.$

and the measured variable is

$$
X_{i}=\sum_{k=1}^{N} x_{i}^{k}
$$

Let us take for simplicity $q=\boldsymbol{P}$. In the previous section we obtained that $\mu$ is proportional to $1 / \boldsymbol{P}$. To avoid leakage of $x_{i}^{k}$ into infinity, let us re-define the form of $f\left(x_{i}^{k}\right)$ in the following way:

$$
x_{i+1}^{k}= \begin{cases}(\mu / P) x_{i}^{k}\left(1-x_{i}^{k}\right), & X_{2}<x_{i}^{k}<X_{1}, \\ a \mathrm{e}^{X_{1}-x_{i}^{k}}, & x_{i}^{k} \geq X_{1}, \\ a \mathrm{e}^{x_{i}^{k}-X_{2}}, & x_{i}^{k} \leq X_{2},\end{cases}
$$

where

$$
X_{1,2}=\frac{\xi \pm \sqrt{\xi^{2}-4 a \xi}}{2 \xi}, \quad \xi=\mu / \boldsymbol{P},
$$


and the parameter $a$ in our calculations is equal to 0.1 (see Fig. 4).

Figure 5 shows the dependence of $\lambda_{1}$ (calculated from the full state vector $\left.\left(x_{1}, x_{2}, \ldots, x_{N}\right)^{\mathrm{T}}\right)$ on $\boldsymbol{P}$. It is more similar to the dependence observed in experiments (there are no sharp peaks and there are similar non-monotonicities in the region of low and high loads).

Thus the generalization of the simple model gives results which are closer to the experimental ones.

The mathematical object proposed in generalization of the model is reasonable to be named "dynamic ensemble with variable structure" (DEVS).

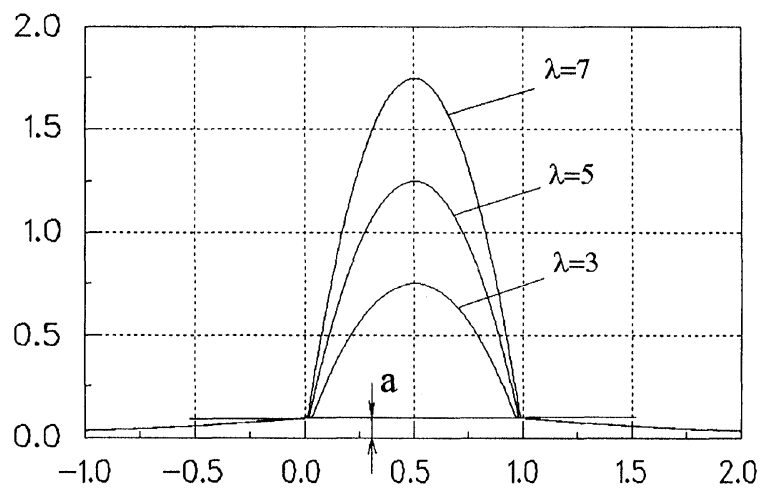

FIGURE 4 Modified logistic map.

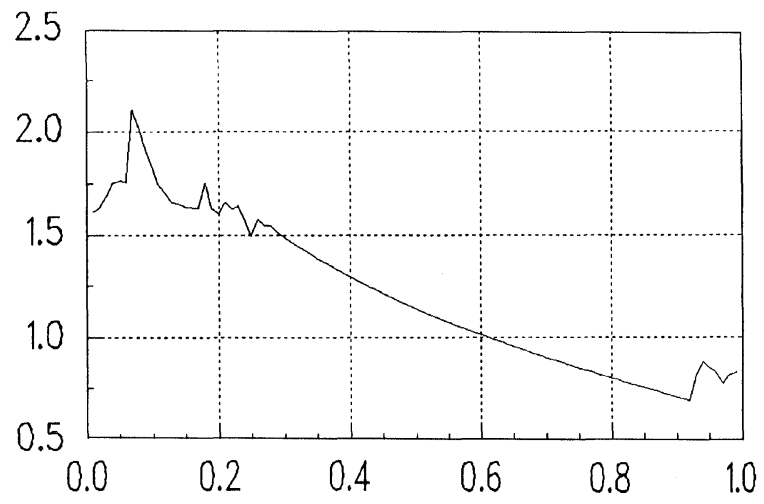

FIGURE 5 The dependence $\lambda_{1}(\boldsymbol{P})$ (from generalized model).

\section{BIFURCATION DIAGRAMS OF DEVS}

Let us consider DEVS which consists of $N$ logistic maps

$$
x_{i+1}^{k}=\left\{\begin{array}{l}
\mu x_{i}^{k}\left(1-x_{i}^{k}\right) \quad \text { with probability } q \\
x_{i}^{k} \quad \text { with probability } 1-q
\end{array}\right.
$$

( $\mu$ does not depend on $q$ ).

(a)

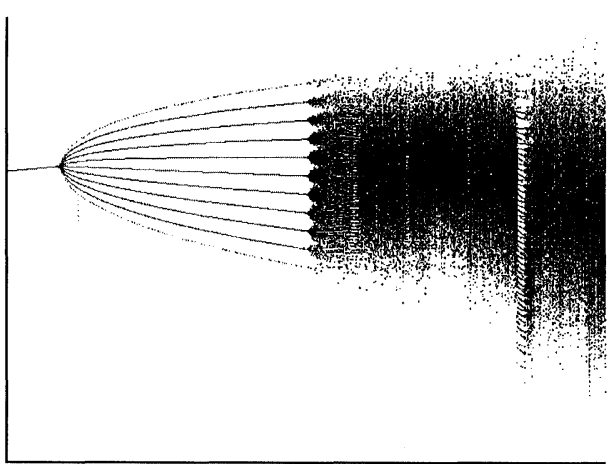

(b)

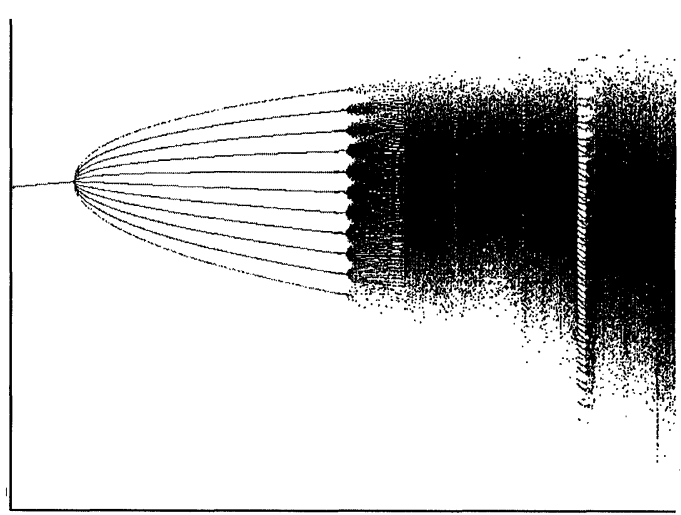

(c)

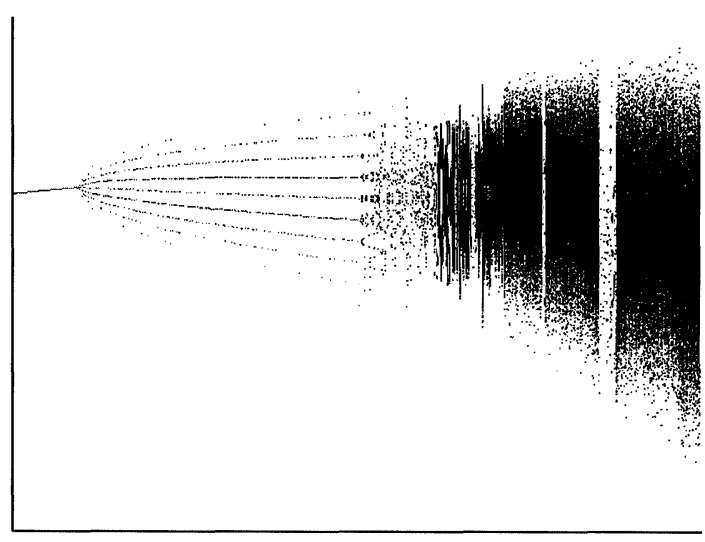

FIGURE 6 Bifurcation diagrams for $N=10$ : (a) $q=0.05$, (b) $q=0.50$, (c) $q=1.00$. 
Figures 6 and 7 show the bifurcation diagrams of DEVS for various values of $q$ and $N$ respectively. With the growth of $q$ the diagrams become more diffuse. After the first bifurcation there are $N+1$ steady states. For one logistic map after the first bifurcation, the "middle" branch of the diagram

(a)

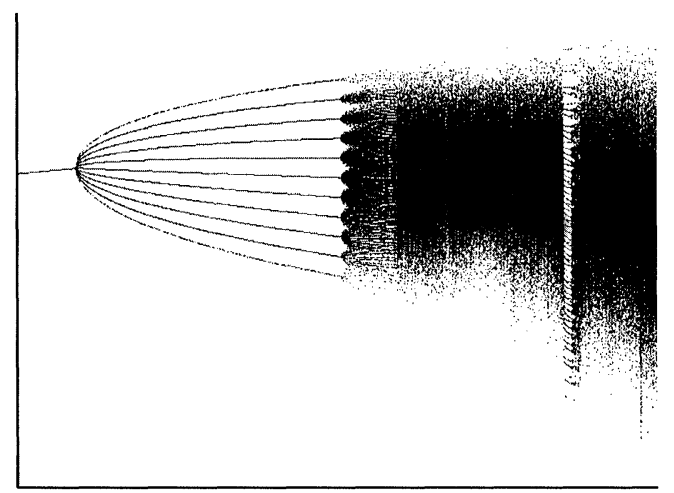

(b)

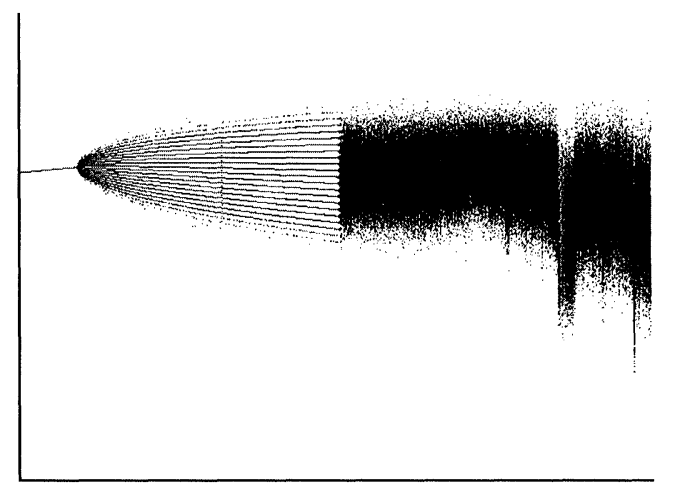

(c)

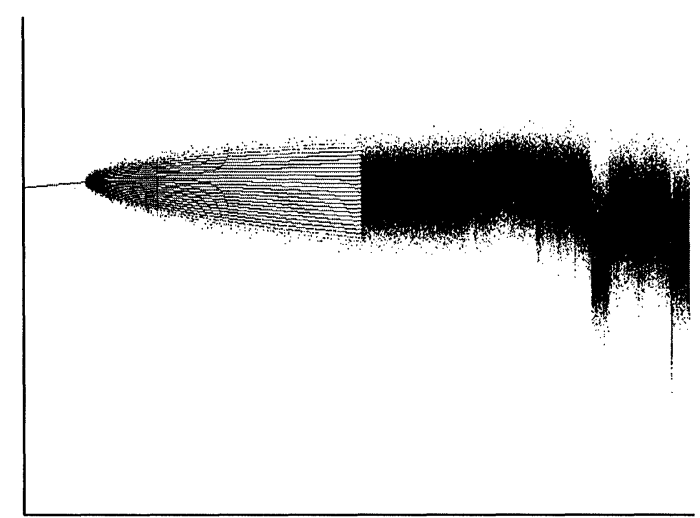

FIGURE 7 Bifurcation diagrams for $q=0.50$ : (a) $N=10$, (b) $N=30$, (c) $N=50$. becomes unstable. If $N=2$ there is stable "middle" branch. Thus if our description of the system is incorrect (say we do not know exactly the number of elements) it is possible to obtain "false" stable branches of the bifurcation diagrams.

Figure 8 shows the mechanical interpretation of this effect. Suppose we consider the elastic beam under the axial load. Figure 8(a) presents the bifurcation diagram and the possible beam configurations. The case of the system of two elastic beams is shown in Fig. 8(b) (the middle line of the

(a)
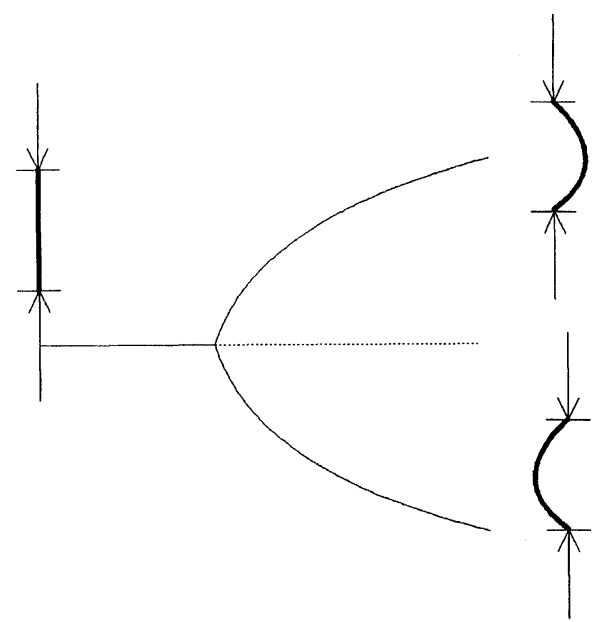

(b)

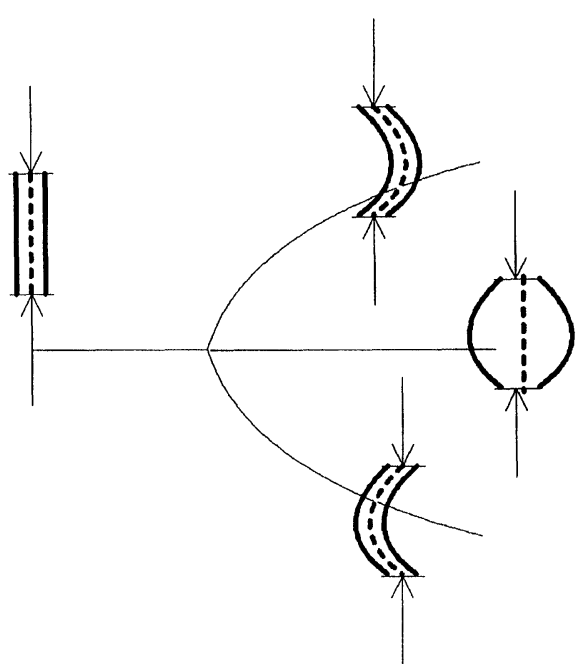

FIGURE 8 Illustration of possible effect in DEVS for $N=2$. 
beams is observable). It is clear that the straight line corresponds not only to the straight (unstable) configuration of the beams, but also to the beams curved in various directions.

\section{CONCLUSION}

The paper presents an approach to the simulation of friction interaction. The model obtained does not use any physical descriptions of the processes in the system, but it has a simple physical interpretation. The generalization of the model gives results very similar to the experimental ones. It is in the form of a dynamic ensemble with variable structure, which has some interesting properties - particularly bifurcation diagrams.

\section{Acknowledgement}

The work was partially supported by Russian Foundation of Basic Research - grant Nos. 9701-00396, 96-01-01161.

\section{References}

Kragelsky, I.V., Dobychin, M.N. and Kombalov, V.S. (1982). Friction and Wear - Calculation Methods. Pergamon Press, Oxford.

Kuzmin, N.N. and Feldstein, I.V. (1996). One approach to tribosystem simulation, Friction and Wear, 3.

Kuzmin, N.N. and Feldstein, I.V. (1997). Chaotic dynamics of friction interaction, Friction and Wear, 6.

Landau, L.D. and Lifshitz, E.M. (1987). Theory of Elasticity. Nauka, Moscow, (in Russian).

Leibovich, H., ed. (1968). Fracture. An Advanced Treatise. Academic Press, New York and London.

Wolf, A., Swift, J., Swinney, H. and Vastano, J. (1985). Determining Lyapunov exponents from a time series. Physica 16D (3) 285-317. 


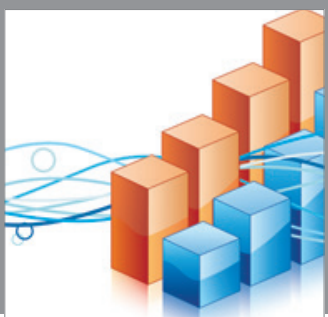

Advances in

Operations Research

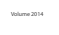

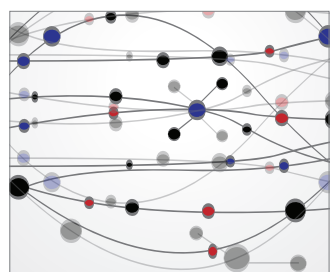

\section{The Scientific} World Journal
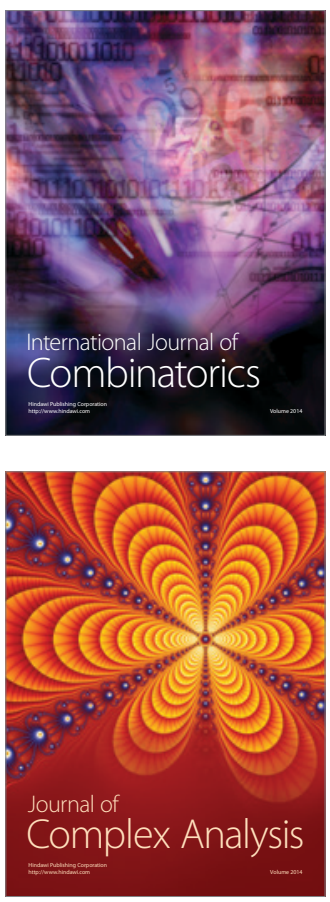

International Journal of

Mathematics and

Mathematical

Sciences
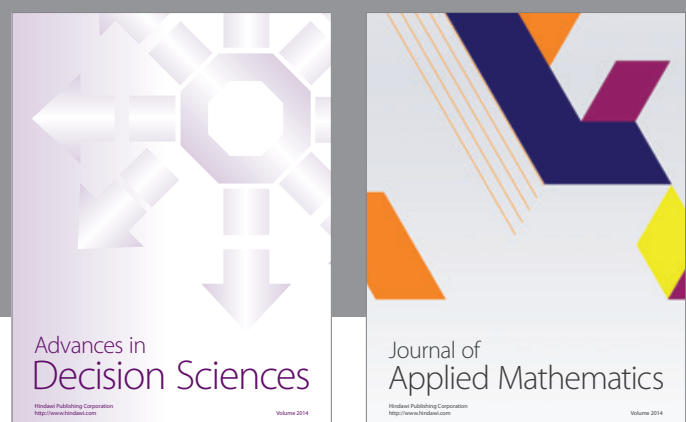

Journal of

Applied Mathematics
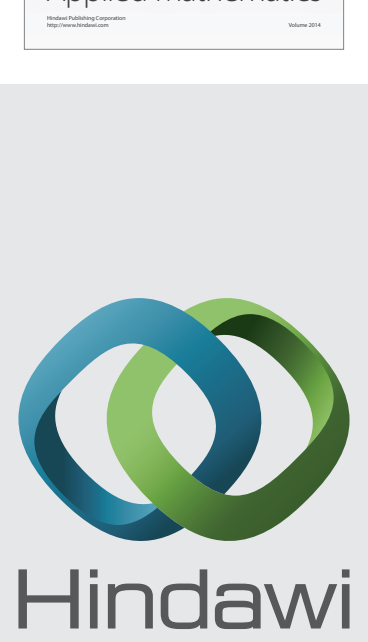

Submit your manuscripts at http://www.hindawi.com
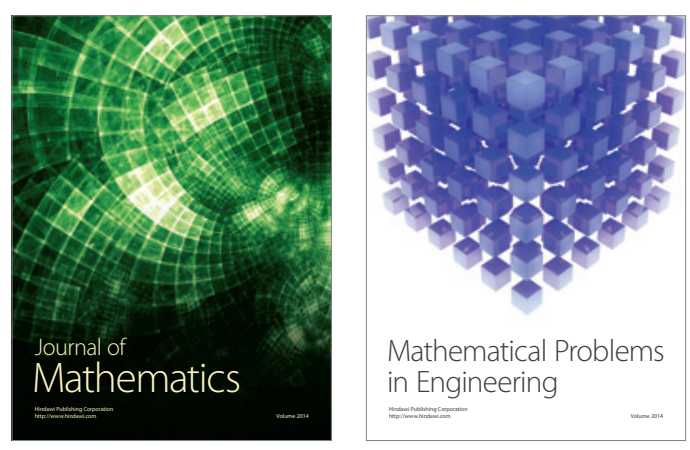

Mathematical Problems in Engineering
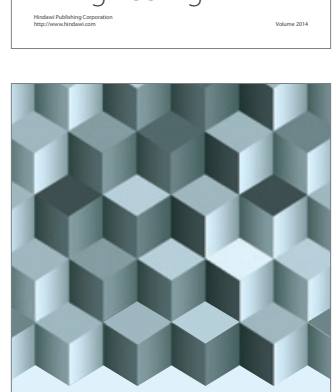

Journal of

Function Spaces
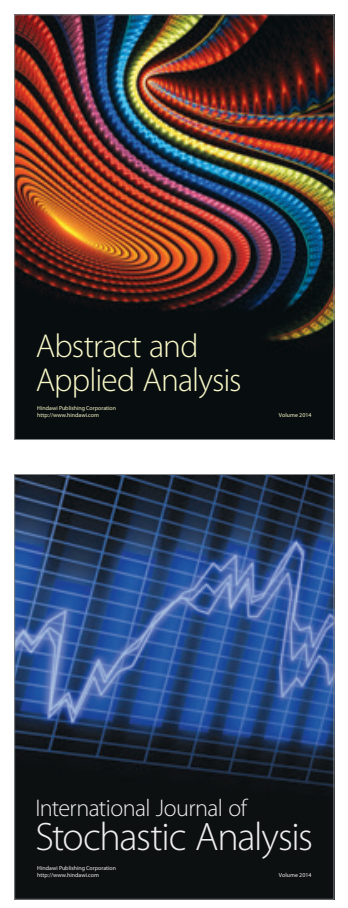

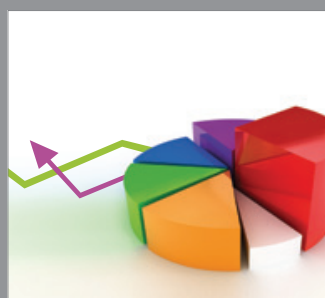

ournal of

Probability and Statistics

Promensencen
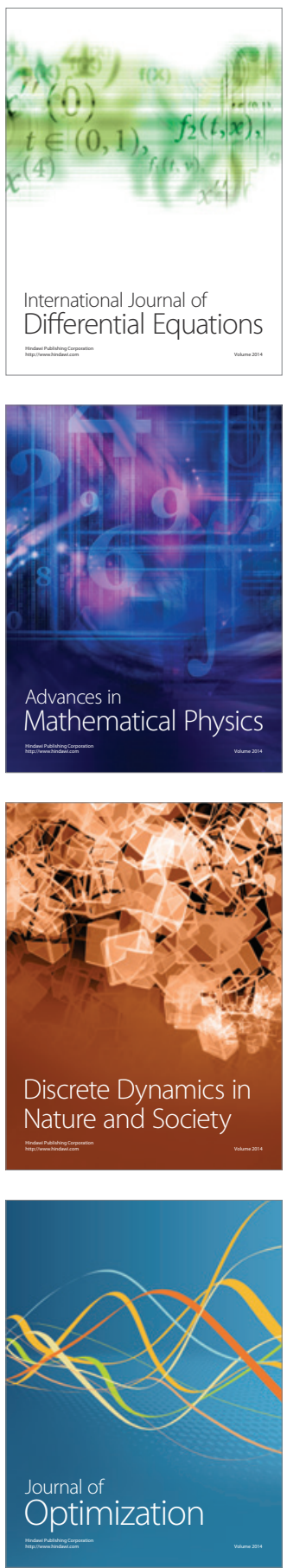